Ueber die schlauchfönnigen Drüsen des Magendarmkanals etc. 375

Fig. 15. Junge Schleimzelle aus dem tiefsten Theil einer Fornix, Starke Vergrösserung. - Eriklärung im Texte.

Fig. 16. Mitose in den oberflächlichen Epithelzellen (Pikrinsäure, Satranin, Alkohol, Damarharz). 250 d. Die Epithelschicht ist schräg durchschnitten; die Lmrisse der einzelnen Epithelzellen sind nicht deutlich zu sehen.

Fig. 17. In Mitosis befindliche Zelle zwischen den Ersatzzellen der tiefen Schicht des Oberflächenepithels (Flemming'sche Lösung, Safranin, Damarharz). 390 d. Das Epithel war etwas schrïg durchsehmitten worden; die Umrisse seiner Zellen sind nicht deutlich zu sehen.

(Aus dem histolog. Laboratorium der I. medizinischen Klinik Prof. v. Koriny i's in Budapest.)

\title{
Studien über den Bau des Chromatins in der sympathischen Ganglienzelle.
}

$$
\text { Von }
$$

\section{Dr. Friedrich Vas}

an der I. medizinischen Klinik.

Hierza Tatel XX.

Die Methode Niss I's ${ }^{1}$ ) zur Darstellung der Chromatinstructur an der Nervenzelle kann man derzeit als die zuverlässigste betrachten. Die Wichlitigkeit dieser. Methode muss anerkannt werden, wenn man bedenkt, dass es bisher unmöglich war, eine scharfe Grenze zwischen physiologischen Zuständen

1) Niss 1, Tageblatt der 58. Versammlung Deutscher Naturforscher und Aerite. 1885. Strassburg. 
und initialen pathologischen Veränderungen der Nervenzelle zu ziehen.

Auffallend ist es, dass sich trotz alledem die normale und pathologische Histologie des Sympathicus dieser. Methode bis nnn nicht bediente, obgleich, wie bekannt, eben dieser Theil der Nervenhistologie einer verlässlichen Grundlage am meisten entbehrt. Wäre diese Methode schon fïiher bekannt gewesen oder derselben in neuerer Zeit eine häufigere Verwendung zu Theil geworden, so wirdle auch bezïglich der physiologischen Berlentung der grossen ,ympathischen (ranglien keine Divergen\% in solchem Maasse herrschen, wie dies bei der Dnrchsicht der diesbezïglichen Fachlitteratur leicht zu constatiren ist. Der Auffassung eines Theiles der Autoren, die in den Ganglien wichtige physiologische Functionen imehabende Gebilde erblicken, steht eine zweite, wie siegmund Mayer sie benemnt, "nililistische" entgegen, welche in den grossen Grenzstrangganglien nur embryologische Reste erkennen will.

Die Untersuchung der Chromatinstructur des Zellleibes der normalen Gauglienzellen scheint geeiguet, dieses Dunkel zn klïren und zur Erkemung der $n$ orma len, functionirenden Zelle eine reelle Basis, untrïgliche differential diagnostische Momente, zu bieten.

Die in geringer Zahl bis num erschienenen Berichte ${ }^{1}$ ) ïber den Werth der $\mathrm{N}$ issl'schen Methode beziehen sich sowohl auf normale Zustainde, als auch auf einzelne pathologische Erscheinungen der $R \ddot{c} \mathrm{ckenmarks-und} \mathrm{(rosshi.rn-Zellen.} \mathrm{Die}$ erwähnten Untersuchungen stellten fest, dass die normale Nervenzelle der grauen Substanz eine typische Formation des Chromatins besitzt, welche schon zu Beginn einer Erkrinkung der Zelle eine leicht zn erkennende Verinderung erführt. Der Grad der Veränlerungen hält stetig Schritt mit dem Grade der Erkrankung, um schliesslich zur totalen Destruction des Chromatins zu führen. Natürlich war das Erkennen diesèr mancherorts sehr sublimen Veränderungen nur so möglich, nachdem vorher die

1) Nissl, l. c. - Fried mann, Ueber progress. Verianderungen der Ganglienzellen etc. Arch. f. Psych. Bd. 19, 1888. - Neurol. Centrablatt 1891, Heft 1. - Schaffer, Neurolog. Centralblatt X. S a r b o, Ungarisches Archir tür Mediz. Be. I, Nr. 4. 
normale Chromatinstructur mit Hilfe der $\mathrm{N}$ iss l'schen Methorle in ihren Details genau erkannt war. Beim Studium der Histologie der Sympathicus-Granglien des Grenzstranges muss nun erst dieser erste Schritt gethan werden. Die vorliegende Arbeit befasst sich auf vergleichender Basis mit der Feststellung der normalen Chromatinstructur des Zelleibes der sympathischen Ganglienzellen.

Die Untersuchungsmethode, die \%ur Erlangung nachstehender Resultate befolgt wurde, war, wie erwälnt, jene Nissl's. Das aus dem eben getödteten Thiere oder aber dem menschlichen Cadaver entnommene Partikelchen . eines Grenzstrang-(xanglions wurde in absoluten Alkohol eingelegt, hicrauf in Celloidin eingebettet. Das in sehr feine Schnitte zerlegte Material kam dann in eine concentrirte wässerige Lösung von Magentaroth (Grïbler), worin die Schnitte entweder einige Stunden hindurch verblieben orler behnfs Beschleunigung der Tinction fiur einige Minuten in der Lüsung ein wenig erwärmt wurden. Hicrauf Auswaschen des iuberschüssigen Farbstoffes in absolutem Alkohol. Den Rest des ïberschüssigen Farbstoffes entzog das Nelkenöl, wohin diu Schnitte schliesslich geriethen. Die Conservirung gesehah in Canadabalsam. Bei der Untersuchung der Chromatinstructur des Zellleibes ist eine gute Oelimmersion unenthehrlich. Die beigegebenen Zeichnungen sind bei einer etwa tausendfachen Vergrösserung (3 mm homogene Immersion und Compensationsocular Nr. 12, Z e is s) gezeichnet. Für die beigeschIossenen Ahbildungen schulde ich Herrn Dr. Emanuel Liebermann aufrichtigsten Dank.

Wie schon früher erwähnt, wurden die Untersuchungen auf Grund vergleichender Basis vorgenommen. Dies geschah auch desshalb, um mit der Richtigkeit einer Auffassung im Reinen $z u$ sein, welche in letzterer Zeit von cinem engrlischen Antor, Namens $\mathrm{H}$ a le $\mathrm{Wh}$ it $\mathrm{e}^{1}$ ), auf Grund eigener Untersuchungen dahingestellt wurde. Der genannte Autor behauptet nämlich, die Grenzstrang-(Hals-)Ganglien der Thi e re wären physiologisch intacte, functionirende Gebilde, während jene des Affen and Menschen nur den Werth embryologiseher Reste hätten. Auf

1) White, On the histology and function of the mammolian superior cerv. gangl. Journ. of phys. VIII, pag. 66 . - White, Further observ, of the histology and function etc. Journ. of phys. X, pag. 341-357. - White, About the phys. and path. value of the Gasserian etc. ganglia. Brain 1890 antumn part. 
diese Auffassung mnss ich noch nach Wiedergabe nachstehender Untersuchungsergebnisse zurückkehren.

Die vergleichenden Untersuchungen wurden an Kaninchen, Hunden, Pferden, Menschen, und zwar an Foeten, $\mathrm{Ne} \mathrm{ta}$ ehorenen, entwickelten Kindern, an Erwachsenen und $\mathrm{Gr}$ e is en ausgeführt.

Bei den Untersuchungen kamen nicht nur die Ganglien der Halspartie, sondern auch jene des Brustabschnittes in Betracht. Auf die Ganglienzellen, welche in den verschiedtenen parenchymatösen Organen ihren Sitz haben, wurde jedoch bei dieser Gelegenheit keine Rücksicht genommen. Hervorgehoben muss werden, dass nur die Chromatinstructur des Z ellleibes, nicht aber auch die des Zellkernes Gegenstand grenauer Beobachtung bildete.

1) Ich beginne mit der Schilderung der norm a le n Chromatinstructur der Ganglienzellen des $\mathrm{H}$ undes. Das Cluromatin dieser Gauglienzellen unterscheidet sich vor allem durch die Form der einzchen Körner von jenem der Rückenmarkszellen. Während dasselbe bei letzterem stäbchenförmig erseheint, ist es in den Sympathicus-Zellen von $\mathrm{kr}$ e is $\mathrm{r} \mathrm{n} \mathrm{der}$, annäherungsweise runder oder vollkommen unregelmässiger Gestalt. Die Kömchen sind bezẗglich ihrer Grösse und $Z$ ah I in Zelleibe verschieden vertheilt. Es ist nicht schwer, an jeder Zelle eine periphere Zone zu erkennen, wo das Chromatin aus grossen und dichtgelagerten Körnchen zusammengesetzt erseheint. Der Durchmesser dieses Ringes beträgt zumeist ein. Viertheil des ganzen Zellleibes. Ein zweiter, ähnlich geformter $\mathrm{R}$ in $\mathrm{g}$ ist in unmittelbarer Umgebung des Zellkernes sichtbar. Dieser zweite Ring ist jedoch bedeutend schwächer als der äussere, besteht meist aus ein oder höchstens zwei Reihen grobkörnigen Chromatins. Zwischen diesen beiden Ringen bleibt naturlich ein Zwischenraum, der aus feinkürnigem, concentrisch um den Kern geschichteten Chromatin zusammengesetzt ist. Die Schichtung des Chromatins ist jener der Rückenmarkzellen ähnlich. So wie bei letzteren, ist auch bei der sympathischen Nervenzelle leicht $z \mathfrak{u}$ erkennen, dass diese Schichtung dort, wo dieselbe einem Zellfortsatze in die Nähe gelangt, allmählich ihre Anordnung wechselt, indem die Chromatinkörner der Längsachse des Fortsatzes entsprechend gelagert erscheinen. In diesen 
Fortsätzen, welche bloss eine kleine Strecke hindurch in der Sclunittebene des Präparates zu verfolgen sind, zeigt die Grösse und Zahl der Chromatinkörner eine auffallende Abnahme.

Bezüglich der Chromatinstructur des Kernes ist kein bestimmter Typus zu entdecken; auch scheint es, wie ich mich durch vergleichende Untersuchungen überzeugte, dass zur Darstellung des Kernchromatins das F l e m m in g'sche Chromosmiumessigsäure-Gemisch zweckmässiger wäre.

Die eben geschilderten Details der Chromatinstructur beim Hunde zeigen unter normalen Verhältnissen bei körperlieb c ntwickelten Thieren immer gena u denselben Typus. Dieser Umstand griebt augleich Anlass mit wenigen Worten der sogenannten chromophoben und chromophilen Zellen ru gedenken, deren Existenz $\mathrm{Fles} \mathrm{ch}^{1}$ ) und dessen Schulerinnen Koneff and Gittis ${ }^{2}$ ) feststellten. Genannte Lutoren untersuchten die Schnitte der sympathischen Ganglien verschiedener Thiere und fanden, dass im selben Schnitte ein Theil der Zellen den Farbstoff sehr intensiv aufnimmt, ein anderer Theil aber beinahe ungefärbt bleibt. Von der Richtigkeit dieses Unstaudes konnte ich mich auch überzeugen, wenn dic Prüparate nach den meisten üblichen Methoden gefärbt wurden, welche die diffuse Tinction des Zellleibes herbeiführen. Die $\mathrm{N}$ is s l'sche Methode jedoch hat, wie bekannt, jenen Vortheil, nur die Chromatinstructur (die filare Substanz [Flemming]) zu tingiren, während die interfilare vollkommen ungefärbt oder schwach rosafarben erscheint. Demzufolge ist es auch ganz unmöglich, mit Hülfe der $\mathrm{N}$ issl'schen Methode anch auf dessen Umstand Rïicksicht zu nehmen. Dieser Mangel jerloch ist in gewisser Hinsicht ein Vortheil, da dieses Verfahren ohne Rücksicht auf' den schon unter normalen Verhältnissen differenten. chenisehen (chromophoben oder chromophilen) Zustand des Zellleibes jede gesunde Zelle gleichmässig färbt und deren Chromatintextur isolirt darzustellen vermag. (Fig. 2).

1) Bemerkungen über die Structur d. Ganglienzellen. Neurol. Centralblatt 1886. - Structur der Nervenzellen in periph. Ganglien. Neurol. Centralbl. 1886.

2) A un a Gittis, Beitr. zur vergl. Histologie d. periph. Ganglien. Dissert. 1887. - Virch ow-Hirs ch, Berichte 1888. 
2) Die Chromatinstructur der Ganglienzellen des Kan in chens unterscheidet sich in wenigen Momenten von jener des Hundes. Die Unterschiede bestehen einerseits darin, dass die Chromatinkörnchen von geringerem Umfange sind, andererseits, dass die zweite, den Kern umgebende Zone der Körner beim Kaninchen vollkommen fehlt. Der äussere Ring jedoch ist constant und gut ausgeprägt vorhanden. Die Chromatinstructur der Kerne, deren gewöhnlich жwei in einer Zelle vorhanden sind, zeigt nichts Typisches. Der Nucleolus ist inmer intensiv gefärbt, die interfilare Substan\% des Kernes vollkommen ungefärbt, jene des Zellleibes jedoch von schwach rosafarbener Nuance (Fig. 1).

3) Bezüglich der P ferdeganglien fehlte es mir leider an genügendem Material, um mit voller Bestimmtheit den Typus des Chromatins feststellen zu können. Ein Umstand jedoch ist es, der gan\% entschierlen zu erkennen war, nämlich der, dass der Zellleib nebst $\mathrm{dem}$ iiberaus feinkörnigen Chromatin sehr zahlreiche $\mathrm{Pig}$ mentk $\ddot{b} \mathrm{rner}$ enthält, welche meist, in einem Haufen zusammengeballt, einen Theil der Zclle einnehmen. Die Wichtigkeit dieses Umstandes wird in den nachfolgenden Zeilen dort crörtert werden, wo der Bedeutung des Pigmentes für das Nervensystem Erwähnung gethan werden muss.

4) Die Untersuchung der Ganglienzellen des Menschen wurde vorgenommen: a) an einem Foetus vom VII. Mon a te der Schwangerschaft. Vergleicht man das hier gewonnene Bild der Ganglienzellen mit jenen des Hundes, ist der Untersehied ein höchst augenfälliger. Die Zellen sind klein, kaum ein Viertel des Durchmessers einer Ganglienzelle, wie man sie im Durchschnitte beim Hunde finclet. Der Zellleib umfasst als schmaler Gürtel den relativ sehr voluminösen Kern. Das Paraplasma oder die interfilare Substanz ist a usgeprägt rosafarben gefärbt. Im Zellleib ist keine Spur einer Chromatinstructur zu finden, derselbe ist vollkommen homogen. Der Kern dagegen ist reich an Chromatinsubstanz (Fig. 3). b) Bei rollkommen entwickelten Neugeborenen vom IX. Monate der $\mathrm{Schwangerschaft} \mathrm{ändert} \mathrm{sich} \mathrm{dieses} \mathrm{Bild.} \mathrm{Die} \mathrm{Zellen} \mathrm{sind}$ beträchtlich, beiläufig um ein Drittel grösser, als die des Foetus; der Zellleib ist ziemlich breit, während das Volumen des Kernes eine leicht erkenubare Reduction erfährt. Das Paraplasma er- 
Strdien über d. Bau des Chromatins in d. sympath. Ganglienzelle. 381

scheint blass rosa gefürbt. Der wichtigste Untersehied aher besteht darin, dass deutliche Spuren des im Entwickeln be rriffenen Chromatins zu erkennen sind. An der Peripherie der Zelle erscheinen namlich die hier und da ziemlich grossen Körnchen, welche sich in einzelnen Zellen zu einem leicht erkennbaren $\mathrm{Kranz}$ formiren. Allenfalls ist ein anderer grosser Theil der Zellen noch frei jeglichen Chromatins. Ein zweiter Umstand, dessen hier nur kurz Erwähnung gethan werlen soll, ist das Erscheinen des gelben Farbstoffes, welcher entweder in feinen diffus zerstreuten Körnern oder in gröberen Klümpchen an einer Seite der Zelle angehäuft erscheint. Jene Zellen, wo das Auftreten dieses Pigmentes zu erkemen war, zeigten bezilglich Chromatinstructur nichts, was dieselben von den farbstofflosen Zellen unterschied.en hätte (Fig. 4). c) Im vorgeschrittenen $\mathrm{K}$ indes a l ter (10-12 Jahre) ist der Entwickelungsgrad der Zellen, was dercn Grösse und Chromatinstructur anbelangt, ein noch höherer. Die Zellen erlangen eime bedentende Grösse. Der Kern ist relativ klein. Der Zellleib weist einen vollkommen entwickelten Typus der Chromatinstructur auf. Der Aufban derselben unterseheidet sich kaum von jenen des Hundes oder Kaninchens: an der Peripherie der Z ëlle ein gut entwickelter grobkörniger Ring, der nach innen allmählich in feinkörniges, concentriseh geschichtetes Chromatin übergeht. In der Nähe des Kernes, dessen Contouren scharf ausgeprägt erscheinen, sind mancherorts einige grössere Chromatinkörnchen zn erblicken. Der Kern ist arm an Chromatin. Der Nucleolus ist intensiv gefärbt. Die Anwesenheit des gelben Pigmentes ist hier noch um vieles häufiger constatirbar. Die meisten Zellen enthalten dasselbe in grösserem oder geringerem Maasse, entweder in Form kleiner Körnchen, welche einen beschränkten Raum in der Zelle einnehmen, oder diffus im ganzen Zellleibe zerstreut sind (Fig. 5). d) Bei körperlieh vollkommen entwickelten Individuen im Alter von 20-40 Jahren zeigt die Entwickelung der Zellen im Allgemeinen, und speriell was das Chromatin betrifft, keine besonderen Fortschritte. Die Grösse der Zellen ist ziemlich dieselbe wie jene der Kinder im Alter von 10 bis 12 Jahren. Das Chromatin jedoch ist reichlicher, der äussere 
Kranz desselben von grösserem Breitendurchmesser und auch das feiner gekörnte Chromatin im Inneren der Zelle dichter gereiht. Ein Unterschied mehr quantitativer Natur bezieht sich anch anf das Z e lle n pigment. Dasselbe ist hier in den meisten Zellen vorhanden und erscheint entweder geformt (gekörnt), oder diffus im Zellleibe imbibirt. Doch muss auch hier ausdrûcklich betont werden, dass die Chromatinstructur dieser stark pigmentirten Zellen vollkommen intact, in jeder Be\% iehum it jener der nichtpigmentirtenZellen übcreinst immend, erschien (Fig. 6).

Bei Betrachtung dieses Umstandes muss ich grleichzeitig jencr Auffassung gedenken, welche, in dem Vorhergehenden schon kurz erwähnt, einem englisehen Autor Namens Hale White ${ }^{1}$ ) entstammt. White fand nämlich, gelegentlich der Untersuchung der Grenzstrang-Ganglien verschiedener Thierarten, dass die HalsGanglien des Affen sowic des erwachsenen Menschen in ihren Zellen viel Pigment enthielten, während jene der iibrigen Thicrspecies, sowie dic der menschlichen F o e te n von Pigment frei waren. Genannter Autor fand auch, dass den Zellen der menschlichen Hals-fianglien. auch - sonstige Zeichen der Destruction anhaften: Zerfall des Protoplasmas, Verfettung, Schrumpfung. White fand gleichzeitig, lass die Ganglienzellen des Herzens der Brustganglien, des Ganglion Gasseri vom Pigment frei und auch sonst intact waren und zwar nicht nur beim menschlichen Foetus, sondern selbst im Erwachsenen. Bei Zusammenfassung seiner Untersuchungsresultate kommt er zu jenem Resultate, dass die Halsganglien des Menschen, nach der Geburt cine stetige regressive Metamorphose eingehen, wodurch dieselben ihre physiologischen Functionen verlieren und schliesslich im Erwachsenen nur den Werth embryonaler Reste beibehalten, welche aus diesem Grunde die erwähnte starke Pigmentation aufweisen. Die Halsganglien aller tibrigen Thierarten jedoch, da deren Zellen pigmentlos und auch sonst intact erscheinen, müssen als stetig functionirencle Organe betrachtet werden. In einer von mir erschienenen früheren Arbeit ${ }^{2}$ ) bemühte ich mich, jene

1) l. c.

2).F. V as, Ueber die phys. Bedeutung der Ganglien im symp. Grenzstrange. Allg. Wiener Med. Z. 1891. 
Bedenken anzufïhren, welche schon a priori beim Durchlesen der Arbeit White's im Leser gerechterweise entstehen. Durch Wiedergabe einiger Untersuehungsergebnisse, die mir schon seinerzeit für dieses Thema zur Verfïgung standen, trachtete ich den angeführten Bedenken eine reelle Basis zu geben. In wiè ferne mein damals eingenommener Standpunkt berechtigt war, baben die seit jener Zeit fortgesetzten Untersuchungen gezeigt nnd sehen wir nun, wie sich die Auffassung White's zu den bis jetzt beschriebenen Untersuchungsergebnissen verhält.

Dass die Anwesenheit des Pigmentes in den Ganglienzellen der menschlichen Foeten nicht $z u$ constatiren ist, ist Thatsache. Thatsache ist ferner, dass das Pigment in den Halsganglien des Erwachsenen in ziemlicher Menge vorhanden, während dasselbe in den Brustganglien spärlich zu finden ist. Nicht zu leugnen ist auch, dass bei zahlreichen Thierspecies das Pigment in den Zellen überhaupt nicht erscheint. Doch wäre ich der Meinung, dass diese Umstände durch andere in ilırer Bedeutung aufgewogen werden. Wic erwähnt, fand sich in den Hals-Gangrienzellen des Pferdes Piginent in grosser Menge vor. Hier kimn auch noch erwähnt werden, dass die Hals-Ganglienzellen des Frosches reichlich Pigment enthalten. Und doch zühlt selbst $\mathrm{W}$ h i te diese Thiere $z$ jenen Klassen, deren Hals-Ganglien ihre Funetion nicht verlieren. Wichtig ist es anch, dass das Erscheinen des Pigmentes auch beim Menschen schon in den ersten Lebensmonaten zu erkennen ist. Dáss übrigens $\mathrm{nicht} n \mathrm{ur}$ die Ganglienzellen des Grenzstranges Farbstoff enthalten, ist eine bekannte Thatsache. Varagli a ${ }^{1}$ ) fand, um ein Beispiel zu erwähnen, einen Theil der Nervenzellen des menschlichen Rü ckenmarkes reich an Pigment, ohne an denselben Merkmale der Atrophie zn erkennen. Eine ganz gewöhnliche Erfahrung ist es ferner, dass einzelne Ganglienzellen des menschlichen Rückenmarkes pigmentirt sind, ohne sonstige Deformation zu zeigen.

Aus all dem ergiebt sich, das das Erscheinen des Pigmentes eine specifische Eigenschaft mancher

1) Varaglia, Sur le pigment de la colonne cellulaire des nerfs mixtes. Arch. Italien. de biologie T. IX. Hoffmann-Schwalbe, Jahresb. Bd. 16. 
Thierspecien ist, ohne dass daras die Erkrankung oder Atrophie der Nervenzellen deducirt werden könnte.

Meines Wissens hat White die Chromatinstructur nicht mutersucht. Denn hätte er dies gethan, so würde er $\%$ jenem Ergebnisse gekommen sein, dass gerade die foetalen, pigmentfreien $\mathrm{Nervenzellen}$ es sind, welche keine Chromatinstructur besitzen, während die am reichsten pigmentirten eine ausgesprochene Textur desselben a $\mathrm{f}$ weisen. Hieraus ist nicht ru sehwer jene Consequenz zu ziehen, dass die foetalen chromatinlosen Zellen es sind, welehe physiologische Functionen noch nicht besitzen und dass mit dem Fortsehreiten der Entwickelung des ganzen Organimus auch die sympatische Nervenzclle, ilurer körperlichen Vollendung entgegenschreitend, e h romatin- und zugleich pigmenthaltig wird. Hierdureherhät ugleich jene Supposition ihre Basis, dass diese Nervenzellen es sind, welche auf Grund ihrer morplologischen Beschaffenheit (Chromatinstructur) auf physiologische Functionen Anspruch er heben können.

e) Wie erwähnt, wurden die Untersuchungen auch anf Ganglien solcher Greise erstreckt, an denen die Section auch sonstige Zeichen des senilen Marasmus aufdeckte. Cnd hier fanden sich thatsächlich Zeichen, welche einer morphologischen Destruction gleich kommen. Die Beschreihung dieser Veränderungen gehört zwar, strengc genommen, in jenen zweiten Theil dieser Abhandlung, welcher die pathologischen Veränderungen der sympathischen Ganglien besprechen soll, doch möge schon hier erwähnt werden, dass die Destruction der Chromatinstructur die Anfmerksamkeit des Untersuchers anf sich lenkt. Die Destruction macht sich in mehreren Formen sichtbar. Die gewöhnlichste Form ist der fein körnige Zerfall derselben, wodurch die Zelle so erseheint, als ob sie fein mit Staub belegt wäre. Ein zweites Stadium macht sich im $\mathrm{Homogenwerden} \mathrm{der}$ Zellsubstanz bemerkbar. Diese Form beginnt in der Mitte der Zelle und scheint gegen die Peripherie vorzuschreiten. Ein drittes Starlium ist ferner an jenen Zellen $z u$ unterscheiden, wo der Zellleib zu einer stark gefärbten, formlosen Masse 
Studien über d. Bau des Chromatins in d. sympath. Ganglienzelle. 385

verwandelt erscheint, welche durch ihre dunkle Färbung die Anfmerksamkeit auf sich lenkt. - Hier und da bemerkt man an Zellen, welche eigentlich als solche nur zu vermuthen, nicht aber zu erkennen sind, zahlreiche grobe und feine Bröckelchen, welche auf einen Haufen zusammengeballt stehen, und den körnigen Zerfall, den letzten Grad vor dem vollkommenen Untergang der Zelle markiren. - Ohne bei diesem Capitel hier länger verweilen zu wollen, möge nur auf die Aelınlichkeit dieser Veriinderungen hingewiesen werden, welche dieselben mit dem von Friedman ${ }^{1}$ ) beschriebenen und bei der Myelitis gefundenen Teränderungen haben (Fig. 7).

Ueberblicken wir die bisherigen Untersuchungsergebnisse, so ist daraus ersichtlich:

1) Dass das Chromatin der sympathisehen Nervenzellen einen strengen Typus einhält.

2) Dass die Entwickelung des Chromatins mit. der allgemeinen körperlichen Entwickelnng des Organismus und derspeciellen Entwickelnng der Nervenzelle Schritt hält.

3) Dass das Pigment eine specielle Eigenschaft einzelner Thicrspecies bildet, olne dass demselben eincbesondere Bedeutung zuzuschreiben wäre.

4) Dass das Chromatin der Nervenzellen des Menschen im Greisenalter eine gewisse Destruction erleidet.

II.

Eine in mannigfacher Beziehung wichtige Frage ist die, ob der Erregungsustand der Nervenzelle durch bestimmte characteristische morphologische Erscheinungen zm Geltung kommt, oder ob überhaupt die gereizte Zelle von der r u benden zu unterscheiden wäre? Nimmt man die bisherigen Erfahrungen, welche man beim Studium des Protoplasmas im Allgemeinen machte, zum Ausgangspunkte, ist obige Frage mit grösster Wahrscheinlichkeit bejahend zu beantworten, da doch bekanntermaassen die Structur des Protoplasmas der Amoeben, der rothen Blutkörperchen etc., anf thermische, electrische, me-

1) Neurolog. Centralbl. 1891, Nr. 1. 
chanische und chemische Reize mit Formveränderung reagirt. Bezüglich der $\mathrm{Nervenzellen}$ jedoch stehen nus nur wenige experimentelle Daten zur Verfüguug und diese wenigen in mancher Beziehung widersprechend, wären kaum geeignet, die ohgenannte Frage einer entgültigen Lösung entgegen zu führen.

Fleis $\mathrm{chl}^{1}$ ) fand im Ganglion Gasseri des Frosches, nachdem er dasselbe in concentrirte Borsäurelösung gethan, dass nebst den Veränderungen, welche der Zellleib in Folge chemischer Einwirkung erlitt, in gewisser Beziehung Erscheinungen aufwies, welche nicht rein chemischer, sondern physiologischer Natur sein mussten; er sah nämlich in zahlreichen Fïllen das A ustreten der Zellkerne aus dem Zellleibe.

Hier möge auch einer interessanten Erfahrung Swierc\%ewsky's und 'Toms a's $\left.{ }^{2}\right)$ Erwähnung gethan werlen. Genannte Autoren sahen an raseh isolirten Ganglienzellen des Frosches die Nucleolen in stetiger Eigenbewegung. Diese Bewegung war eine ziemlich rasche und durch Hinzuleitung von versehiedenen Gasen die Schnelligkeit derselben beeinflussbar.

Korybutt-1)askievits ${ }^{3}$ reizte den Ischiadicus des Frosches electrisch whd unterstichte hernach jenen Theil des Ruickenmarkes, ans dem die Wurzeln des Ischiadicus entspringen. $\mathrm{Er}$ fand als auffalligstes Symptom die bedentende Volumzunahme ler Zellkerne.

$\mathrm{Hod} \mathrm{g}^{4}$ ) jedoch kam zu einem ganz entgegengesetzten Resultate. Er reiıte nämlich mittelst schwachem, indncirtem Strome die einem unterspinalem Ganglion entspringenden Nervenbündel einige Stunden hindurch und fand nachher die Zellkerne geschrumpft und von stärker gekörntem A u s s e hen. Diese Veränderungen waren nach etwa 24 stündiger Ruhe verschwunden.

Meines Wissens wäre dies das Resumé der bisherigen Untersuchungen, welche sich auf den Erregungszustand der Nervenzellen beziehen.

Die Methode $\mathrm{N}$ issl's scheint naturgemäss dazn geeignet,

1). Berichte der Wiener Akad. d. Wissensch. 1873.

2) Zur Phys, des Kernes und Kernkörperchens der Nervenzellen des Sympathicus. Centrilbl. 1869.

3) Arch. f. mikrosk. Anatomie Bd. 32.

4) Centralbl. tüir Physs. III, 1889 und 1891, Bd. V. 
Studien über d. Bau des Chromatins in d. sympath. Ganglienzelle. 387

auch in dieser Beziehung. verwendbar zu sein, da aus den Untersuchungen $\mathrm{H}$ e it z m an n's hervorgeht, dass das Protoplasmageriist also die eigentliche Chromatinstructur in Folge der angewandten Reize Formveränderungen aufweist. Dic diesbezüglichen Experimente nahm ich an Kaninchen vor. Der freigelegte Grenzstrang des Halsabschnittes wurde unterhalb des Ganglions supremum mittelst inducirtem Strome gereizt. Die Electroden jedoch wurden $3 \mathrm{~cm}$ abwärts vom Ganglion supremum nebeneinander anfgelegt. Diese Cautele schien deshalb nothwendig, da sonst die eventuell auftretenden Veränderungen der Zellen leicht einer meehanischen Einwirkung des Stromes eingerechnet werden könnten. Der Rollenabstand betrug $15 \mathrm{~cm}$, die Dauer der Reizmng 15 Minnten. Auffallend war schon während des Experimentes die $R$ io th $\mathbf{u} u g$ und T u res ce ns des Ganglions supremum, welche schon nach etwa 5 Minuten eintrat und bis zur Beendigung des Reizes stetig zunahm.

Nach 15 Minuten wurde das Ganglion beiderseits vom Grenzstrange rasch abgetrennt und in absoluten Alcohol oder in concentrirte Sublimatlösung gebracht. Behuts Controlle und um eine sichere Basis des Vergleiches zu besitzen, wurde das Ganglion supremum der nicht gereizten Seite derselben Procedur unterworfen und gleichzeitig untersucht. Die Experimente wurden in derselben Weise in 6 Fallen wiederholt ${ }^{1}$ ).

Es drängt sich schon hier die Frage anf, ob das Einlegen der Ganglien in absoluten Alcohol, deren morphologische Eigenschaften nicht dermaassen verändert und so zahlreiche Kunstprodncte schafft, dass die Beurtheilung der natürlichen Verhältnisse erschwert oder ganz unmöglich wird? Diese Frage, zweifelsohne von grosser Wichtigkeit, bemühte sich schon $\mathrm{Pec} q \mathrm{q}$ e $\mathrm{ur}^{2}$ ) in's Klare $\mathrm{zl}$ bringen, indem er Ganglien den verschiedensten tiblichen Fixirungsmethoden unterwarf, um die Grösse der entstandenen Kunstproducte, welche den verschiedenen Methoden eigen sind, beurtheilen zu können. P e c q u e ur kam zur Ueberzeugung, dass die Alcoholfärbung, insbesondere nach vorhergehender Sublimat-

1) Obengenannte Experimente wurden im phys. Institute der $k$. ungar. thierärztlichen Akademie ausgeführt.

2) P e c q u e ur, Krit. Bemerk. über die Bedeutung der Kunstproducte bei der Beurtheilung ent»ündlicher und atrophischer Processe. Neurolog. Cuntrablbl. 1886, S. 398. 
fixirung, die verlässlichste ist, während die sonst grebräuchlichen Chromsalze die Ganglienzellen am meisten deformiren.

Aehnliche Vergleiche bezilglich des Werthes der ublichen Härtungsmethoden nahm anch ich behufs Orientirung vor, und kann nich taher auch auf Grund eigener Ceberzengung der Auschauung P e c q n e r's anschliessen.

Die gehïrteten Ganglien wurden nachher der $\mathrm{N}$ is s l'sehen Färbungsmethode unterworfen. Der Vergleich der gereizten mit den nicht gerei\%ten Ganglienzellen lieferte in mancher Beriehmo a ufallende Resultate, welchc sich anf den Zellkern bezieluen. Der Kern ist in den gereizten Zellen bedeutend grösser, erscheint wie geschwellt, nebstdem verlïsst er seinen Plat\% im Innern der Zelle und drüngt sich stark peripheriewärts. In manchen Zellen ist diese Tenden\% derart ansgepraigt, dass die Contomre der Zelle durch den nach auswärts drängenden Kern eine leichte Ausbuckelung erfälırt. Nincherorts ist jene eigenthümliche Erscheinmog zu beobacliten, dassein Segment des Zellkernes die Peripluerie der Zelle ï berschreitet. Vollkommen ausgetretene Zell-. kerne jedoch komnten nicht entleckt werlen.

Das Chromatin des Kernes scheint etwas reichlicher, ohne jedoch rlass irgend eine typische Formation erkenubar wäre.

Eine andere Seric von Veränderungen bezicht sich auf den $Z$ ellleib, der um etwa ein Drittheil vergrössert erscheint. Natiulich machen sich die Veränderungen anch an der Formation des Chromatins bemerkbar. In einigen Fiallen waren diese Verïnderungen rlesselben sehr hochgradig und bestanden darin, dass di e Umgebung des Zellkernes an Chromatin aufillend arm wurde, ja sogar vollkommen chromitinlos erschien. Andererseits jedoch machte sich an der Peripherie der Zelle eine Sta u u $\mathrm{n} g$ der Körner bemerkbar, wodurch ein sehr starker und grobkürniger Ring entstand. Anch hier also macht sich die Tendenz des Peripherwärtsschreitens geltend (Fig. 8). - Diese erwähnten Erscheinungen, welche aus bisher unerforschbaren Ursachen einmal sehr ausgeprägt, ein anderesmal jedoch schwächer zum Ausdruck gelangten, waren als Resnltat der electrischen Reizung immer zu erkennen. Dass diese Erscheinungen der meehanischen Wirking des electrisehen Stromes entstammen 
Studien über d. Bau des Chromatins in d. sympath. Ganglienzelle. 389

würden, ist nicht wahrscheinlich, da, wie erwähnt, der inducirte Strom verwendet wurde, and zwar in einer Entfernung, dass die an und für sich geringe mechanische Leistung desselben auf das Ganglion nicht übergreifen konnte. Die Veränderungen selbst, hauptsächlich das Peripherwärtsschreiten der Kerne, geschah den verschiedensten Richtungen nach, während, wenn diese Erscheinung eine physikalische Wirkung der Stromes wäre, auch das Austreten der Kerne immer dieselbe Richtung beibehalten müsste.

Aus alldem geht hervor, dass die beschriebenen Veründerungen der Ganglienzellen dem Reizzustande als dessen characteristische morphologische Eigenschaften " ureschrieben werden müssen. Allenfalls ist es sehr wahrscheinlich, dass unter physiologischen Verhültnissen diese Veränderungen in solchem Grade nicht zur Entwicklung gelangen, da die physiologisch, den Ganglienzellen zukommenden Reize, was Heftigkeit anbelangt, dem experimentell angewandten inducirten Strome nicht gleichzustellen sind.

\section{Erklärung der Abbildungen anf Tafel XX.}

Fig. 1. Schnitt aus dem Gangl. cerv. supr. des Kaninchens (nieht gereizt). Vergröss. 1000. Nissl'sehe Fürbung.

Fig. 2. Schnitt aus dem Gangl. cerv. supr. des Hundes. Vergr. 1000. Nissl'sche Färbung.

Fig. 3. Schnitt aus dem Gangl. cerv. supr. eines menschlichen Foetus vom VII. Monate. Vergr. 1000. Nissl'sche Fiirbung.

Fig. 4. Schnitt aus dem Gangl. cerv. supr. eines Nengeborenen. Vergr. und Färbung wie oben.

Fig. 5. Schnitt aus dem Gangl. cerv. supr. eines 12jahrigen Knaben. Vergr. und Färbung wie oben.

Fig. 6. Schnitt aus dem Gangl. cerv. supr. eines 28 jahrigen Mannes. Vergr. und Fiarbung wie oben.

Fig. 7. Schnitt aus dem Gangl. cerv. supr. eines 70jährigen Mannes. Vergr. und Fürbung wie oben.

Fig. 8. Schnitt aus dem Gangl. cerv. supr. eines Kaninchens, dessen Grenzstrang 15 Minuten mit inducirtem Strome gereizt wurde. Fig. 1 ist der nicht gereizten Seite desselben Thieres entnommen. Vergr. und Fiarbung wie oben. 